\title{
Acromegaly and the information gap: patient perceptions of the journey from primary to tertiary care
}

\author{
Hei Yi Vivian Pak ${ }^{1,2}$, Andrew Lansdown ${ }^{4}$, Peter Taylor ${ }^{3,4}$, Dafydd Aled Rees ${ }^{4,5}$, John Stephen Davies ${ }^{4}$ and \\ Caroline Hayhurst 1 1,2
}

${ }^{1}$ Department of Neurosurgery, University Hospital of Wales, Cardiff, UK

${ }^{2}$ Cardiff University School of Medicine, Cardiff, UK

${ }^{3}$ Thyroid Research Group, Systems Immunity Research Institute, Cardiff University School of Medicine, Cardiff, UK

${ }^{4}$ Centre for Diabetes and Endocrinology, University Hospital of Wales, Cardiff, UK

${ }^{5}$ Neuroscience and Mental Health Research Institute, Cardiff University, Cardiff, UK

Correspondence should be addressed to C Hayhurst: caroline.hayhurst@wales.nhs.uk

\begin{abstract}
Objective: Acromegaly is a rare condition and there is often a long path to diagnosis for many patients. We sought to explore patient's perceptions and understanding of acromegaly, to examine the quality of communication and find gaps in the information provided at diagnosis.

Design: A prospective study using qualitative research methodology and grounded theory. A semi-structured interview was conducted with 18 patients treated for acromegaly in a single tertiary centre and verbatim transcripts were thematically analysed for overarching themes.

Results: Eighteen patients with acromegaly were interviewed. The mean age of participants was 52 (range 30-72). Four overarching themes emerged; (1) Patients rely on online resources to understand acromegaly in the time between diagnosis and tertiary care clinic; (2) There is not enough support available for patients; (3) Patients have a basic understanding of acromegaly and associated conditions, but the long-term impact is underestimated; and (4) Patients initially felt intimidated by the multidisciplinary team panel, but overall found it useful.

Conclusion: Acromegalic patients have a strong need for information at the point of initial diagnosis, in particular online resources and interaction with other experienced patients. Wider dissemination of patient educational resources into primary and secondary care settings may improve overall patient satisfaction, treatment adherence and subsequent health care provider-patient relationships.
\end{abstract}

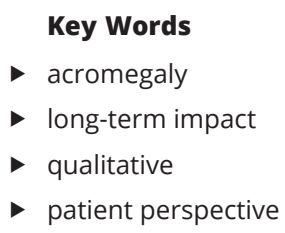

Endocrine Connections (2020) 9, 971-977

\section{Introduction}

Acromegaly, usually due to a benign pituitary adenoma, is a rare condition that has profound effects on all aspects of the body (1). Besides causing soft-tissue growth, excess growth hormone is associated with co-morbidities such as hypertension, cardiomyopathy, diabetes and cancer (2). Acromegaly can be a challenging condition to identify and patients may often be diagnosed late (3). By the time they attend clinic, patients may have already developed significant health problems, with a significant impact on both quality of life and life expectancy $(4,5)$. Additionally, the diagnostic delay often shapes patients' attitudes to health care professionals, which impacts on the quality https://ec.bioscientifica.com

https://doi.org/10.1530/EC-20-0335

C) 2020 The authors Published by Bioscientifica Ltd

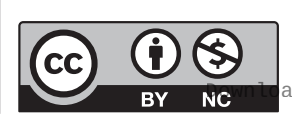

This work is licensed under a Creative Commons Attribution-NonCommercial 4.0 International License. ded from Bioscientifica.com at $04 / 26 / 2023$ 12:42:39PM 
of communication and subsequent treatment adherence $(6,7)$.

In UK practice, often a secondary care endocrinologist or the primary care physician conveys the diagnosis to the patient before referral to a tertiary centre multidisciplinary team (MDT). This model is increasingly the gold standard of pituitary care in North America and Europe $(8,9)$. Therefore, it is anticipated that patients are informed of their diagnosis and given background information prior to their tertiary clinic appointment to discuss treatment options, including pituitary surgery, medical treatment with somatostatin analogues or radiotherapy. However, with an incidence of only 3.8 per million population (10), many primary care and secondary care physicians may lack experience with the condition. Studies of the diagnostic pathway in acromegaly identify the point of diagnosis disclosure as critical in establishing patient-healthcare provider trust $(6,11)$. Additionally, acromegaly patients are often reluctant to share concerns or questions with healthcare providers at initial consultation (6). However, there are few studies focusing on how information should be provided and how information resources are used by patients.

Through qualitative research, this study aims to gain a better appreciation of patients' experiences with the diagnosis of acromegaly. We sought to identify gaps in patient information in the pathway to treatment, in order to improve future communication and to identify the support resources required and when they are needed.

\section{Patients and methods}

\section{Study design}

A prospective qualitative study using a single semistructured interview with patients diagnosed with acromegaly referred to a single regional tertiary pituitary centre.

\section{Participants}

All patients aged over age 18 that had attended the pituitary multidisciplinary pituitary clinic at the University Hospital of Wales with a biochemically confirmed diagnosis of acromegaly were invited for interview by telephone and provided with written information about the study. Interviews were conducted via telephone or face-to-face.

\section{Data collection and analysis}

Data collection and analysis in this qualitative study was based on the grounded theory where ongoing data analysis leads to further questions to refine evolving theories (12). As such interviews are conducted until no further themes emerge and saturation is reached (13). This allowed systematic generation and development of theories by being alert to emerging themes (14), prompting adding new questions to the interview guide. An initial interview guide was developed and subsequent questions added as new themes emerged during the study (Table 1).

Each interview was recorded on tape and transcribed verbatim by the interviewer. Then, the transcripts were re-read to get an impression of the patients' experiences. Thematic analysis was conducted first through open coding, to separate the data into segments which relate to one idea, then though axial coding, to combine similar ideas into overarching themes (15).

Demographic data for each participant were collected, including age, mode of presentation and region of presentation.

\section{Ethical considerations}

This study was approved by the Cardiff and Vale University Health Board Specialist Services clinical board as a service evaluation and all participants provided verbal consent.

\section{Results}

In total, 25 patients were invited for interview. Eighteen patients were interviewed and data saturation was reached as the final interviews did not produce any new themes (16). The mean age of participants was 52 (range 30-72); the mean age at diagnosis was 48 (range 26-72). Table 2 outlines the patient demographics. Eleven patients (61\%) were diagnosed by an endocrinologist in their local hospital, 1 patient received the diagnosis from an orthopaedic surgeon after referral for carpal tunnel syndrome and 4 (22\%) were diagnosed by their primary care physician.

\section{Thematic analysis}

Analysis of the interviews produced four overarching themes:

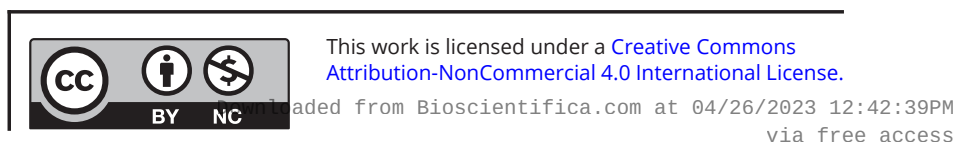


Table 1 Semi-structured interview guide.

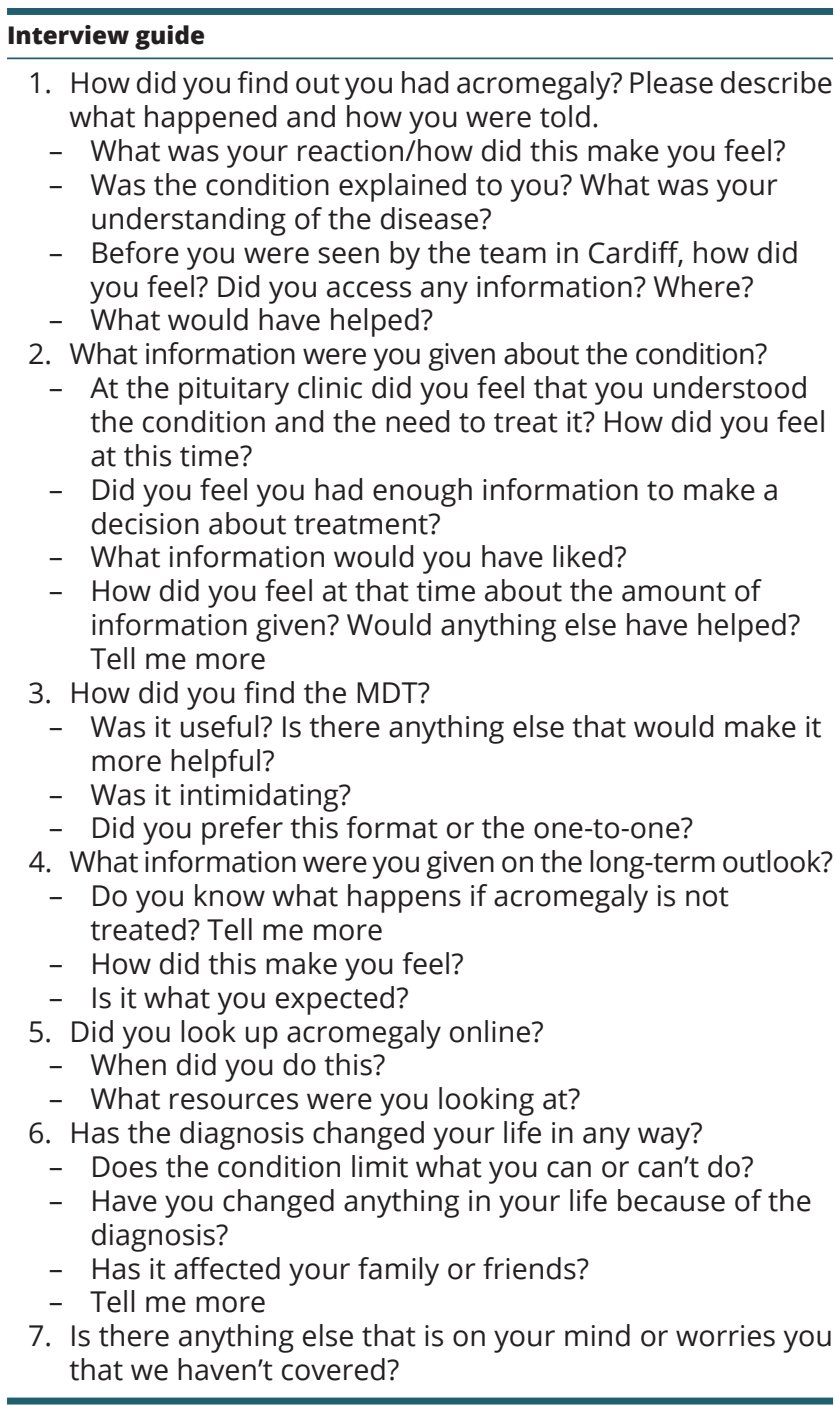

\section{1: Patients rely on online resources to understand acromegaly in the time between diagnosis and tertiary care clinic}

Since acromegaly is a rare disease, patients may be entirely unaware of the condition and hence are surprised when they are given the diagnosis. Most patients felt they were not given enough information at diagnosis, which is usually delivered by their local endocrinologist or general practitioner (GP). Instead, they received the most information from the tertiary MDT clinic, as expected for a rare disorder. Only five patients reported that printed resources would have been useful at diagnosis.

"It wasn't brilliant. There's not a real pamphlet and nothing that was explaining it. It was literally, 'you got acromegaly' ... most of it was done by myself, reading online about it"
"Possibly a leaflet about ... explaining things. And as I say, what the pituitary gland does and the whole ... some of the terminology ... of the various things that were going on"

Given delays between obtaining the diagnosis and attending the tertiary care clinic the greatest information need is between appointments. All patients, except one, had searched online to learn more about acromegaly. All patients who searched online did so as soon as they heard about their diagnosis. However, many reported having to filter out what they read online as there is an overwhelming amount of information available and some can be distressing for patients to see.

"When I googled it and there was very outdated ... there was a man with a gigantic jaw. When you google things, you can get lost"

Many patients used the National Health Service website Health A-Z (www.nhs.uk) to find information on acromegaly. Other resources which patients reported using are online groups such as on Facebook groups, forums, videos, podcasts and blogs. To find information, patients used google with the search terms 'Acromegaly' or 'Pituitary Tumour'. Five patients reported they found a number of North American resources online, for example, websites and video interviews, but few specific to the United Kingdom or their region. Patient videos and websites from the UK may be more relevant and reassuring for the patients in this study.

"I think the ones I remember were NHS UK, also The Pituitary Foundation and there seems to be a lot of academic stuff from America but if I remember rightly I started looking at sort of threads and help groups but it was just full of ... it was the worst stories, you know, so I decided not to read about it anymore. I thought it would help but actually really didn't, so I stuck with the facts, the clinical facts"

When asked if they had enough information to support treatment decisions, the majority of patients felt that initially, they thought surgery was the only option, but learning about the procedure and alternative options helped put them at ease. With information, patients felt confident in the team treatment recommendation.

\section{2: There is not enough support available for patients}

All participants reported they would have liked to receive more support, as GPs are often unable to help with issues relating to acromegaly.

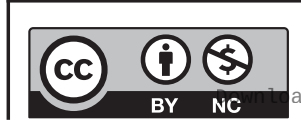

This work is licensed under a Creative Commons Attribution-NonCommercial 4.0 International License. ded from Bioscientifica.com at 04/26/2023 12:42:39PM via free access 
Table 2 Patient demographics.

\begin{tabular}{|c|c|}
\hline Interview no. & Age at interview (years) \\
\hline 1 & 40 \\
\hline 2 & 30 \\
\hline 3 & 60 \\
\hline 4 & 50 \\
\hline 5 & 65 \\
\hline 6 & 31 \\
\hline 7 & 70 \\
\hline 8 & 60 \\
\hline 9 & 33 \\
\hline 10 & 57 \\
\hline 11 & 56 \\
\hline 12 & 32 \\
\hline 13 & 74 \\
\hline 14 & 61 \\
\hline 15 & 63 \\
\hline 16 & 43 \\
\hline 17 & 65 \\
\hline 18 & 40 \\
\hline
\end{tabular}

\begin{tabular}{c}
\hline Age at diagnosis (years) \\
\hline 39 \\
26 \\
58 \\
33 \\
61 \\
29 \\
66 \\
58 \\
31 \\
54 \\
55 \\
31 \\
72 \\
59 \\
59 \\
39 \\
62 \\
31
\end{tabular}

\begin{tabular}{c}
\hline Gender \\
\hline$M$ \\
$F$ \\
$F$ \\
$M$ \\
$M$ \\
$F$ \\
$F$ \\
$F$ \\
$M$ \\
$F$ \\
$F$ \\
$F$ \\
$F$ \\
$F$ \\
$F$ \\
$M$ \\
$M$ \\
$M$
\end{tabular}

Site of diagnosis

Primary care

Local endocrinologist

Local endocrinologist

Local endocrinologist

Local endocrinologist

Orthopaedic surgeon

Primary care

Tertiary endocrinologist

Primary care

Local endocrinologist

Local endocrinologist

Tertiary endocrinologist

Primary care

Local endocrinologist

Local endocrinologist

Local endocrinologist

Local endocrinologist

Local endocrinologist

"Because they know you're under specialists, they can't help you because they just don't know"

Patients find the ability to contact an endocrine nurse specialist for advice reassuring but this is often only available at the tertiary centre, following specialist referral.

"There is an endocrine nurse centre there. Because I know the consultant will get back to you, but he very busy doing wards, doing the clinics, sometimes there is no one else that's in, to offer any advice"

Importantly, patients expressed they would have liked to talk to treated patients, to get a better sense of what is happening and what they are about to go through. Meeting other people with acromegaly and reaching out to support groups could be an invaluable source of support for the patients.

"Maybe meeting other people who has had it and have been cured for it ... so you know what they've been through, what to expect. That would have been helpful"

Earlier signposting in the primary or secondary care setting to online resources and support groups in important in the patient pathway.

"Maybe like The Pituitary Foundation, maybe it would have been best if I would ... been told to ... contact The Pituitary Foundation you know? To chat with them or the pituitary nurse ... that would have been very good actually"
3: Patients have a basic understanding of acromegaly and associated conditions, but the long-term impact is underestimated

Despite feeling they lacked information, the majority of patients were aware acromegaly was associated with a growth hormone-secreting pituitary tumour and softtissue growth. Almost all patients stated there was not enough information given about the long-term outlook of acromegaly, but they were aware of the hormone imbalance and the physical changes that would occur if left untreated. The knowledge of other long-term complications of acromegaly was variable, but the majority of patients were able to name several.

"All I know it is ... it causes the growth hormones to ... grow, which umm ... make my fingers swell up and ... and my feet ... uhh, and obviously part of my face"

"If it's not treated, it can cause shortening of life, umm, you heart can grow and your diabetes could get worse, you can have strokes, you can have a risk of heart attacks"

"Well, the heart problems, the diabetes or the bowel problems, I don't know any more than that"

In the beginning, patients assumed that they would be cured after the surgery and would be normal again. They may not have realised that they will still require follow up for recurrence, and may still suffer from symptoms after surgery, for example, headaches and fatigue. 
"It's just, I can't see an end to it, I don't feel like there will ever be an end to it. You still gotta be checked after. But I can't see a light at the end of the tunnel"

"It wasn't explained that this might not be the end of it, you might need to have another operation and it might not ... I think I was naïve a little bit then, I hadn't realised that"

Acromegaly had a varied long-term impact on patients, $50 \%$ felt the disease had not greatly impacted their lives and were able to get back to normal following surgery. However, for the remainder the experience was life-changing and they still struggle with work and relationships despite successful treatment. Many patients reported ongoing problems with depression and anxiety.

\section{4: Patients initially felt intimidated by the tertiary multidisciplinary clinic, but overall found it useful}

Patients reported being given a minimal warning before attending the MDT of the nature of the clinic. Therefore, some of them felt shocked to see a large group of clinicians and nurses in the room. They were put at ease quickly and have generally benefitted from the team meeting.

"It was useful I guess, you had the input of a lot of different specialists at the same time, so yeah. It was intimidating but it was also reassuring to know that there was ... more than one person looking at your case"

"Well only when you go in and you see seven people sitting there and they're all, you know, professors and consultants ... they were absolutely fine ... but it's a bit intimidating just to see that panel"

Patients benefit from meeting the whole team that will guide them through their treatment and follow-up, however, providing information prior to the clinic on the team members and roles would be useful.

\section{Discussion}

Using qualitative research methods to explore the needs of patients newly diagnosed with acromegaly in a primary or secondary care setting demonstrates the need for high-quality, relevant online resources and local support networks. The internet is being used increasingly by patients to research their health conditions $(17,18)$. This has been shown to affect the patient's beliefs and potentially change their decision about treatment (19). Our study shows that patients diagnosed with acromegaly turn to online resources early after initial diagnosis to learn more about their condition. Interestingly, patients appear much more reliant on online information and patient-to-patient interaction than the traditional printed information leaflet.

However, patients may be overwhelmed by what they might find online and, quality of information on the internet may be substandard (20). Our cohort highlighted the importance of online materials and groups being regionally specific to them. Ideally, patients would like to be provided with reliable online resources at initial diagnosis and guidance to help them make sense of the information (21). In a similar study by Gurel et al. (6), 19 patients participated in online and face-toface interviews aimed at understanding the impact of a diagnosis of acromegaly. They demonstrated a strong desire for education about the disease at diagnosis, noting the diagnosis seemed to 'fuel a thirst for knowledge' in all participants (6). As in our study, participants emphasised the need for patient-patient interaction as part of their quest for knowledge and a desire to take control of their disease, highlighting the need for access to support groups. Plunkett and Barkan (11) suggest strategies to facilitate the patient-healthcare professional relationship throughout the treatment pathway and highlight the provision of educational and emotional support resources at the initial diagnosis meeting. Such resources include The Pituitary Foundation (www. pituitary.org.uk). In particular, their support groups and peer support programme may address the patients' needs to speak with more experienced acromegalic patients and encourages the exchange of health information $(18,20,21)$. Video presentations by similar patients, such as the UK acromegaly meetup, could be helpful for newly diagnosed patients (UK Acromegaly Meetup 2017: Patient stories - Rachel and Carolyn; https://youtu.be/ llqhT-FheMA).

Internationally, the Pituitary Society (www. pituitarysociety.org), acromunity.com and AcromegalyCommunity.com provide resources and access to support groups. However, in a study to assess communication practices among endocrinologists, Polanco-Briceno et al. (22) reported only $14 \%$ of respondents routinely recommended educational resources or programs to patients and only $44 \%$ were aware of these resources. Additionally, most physicians in the study did not have a dedicated nurse to discuss these topics with patients.

Qualitative research is becoming more popular in surgical and medical practice (23), as it can provide

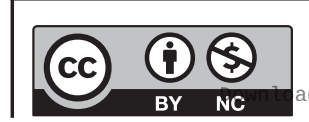


an insight into the social aspects of being treated for a disease from the patient's perspective (24). To date, most qualitative research in acromegaly focuses on diagnostic delay and treatment adherence $(7,25)$. A study by Sibeoni et al. (7) conducted with 18 participants revealed the lack of awareness in the medical community as a significant factor for a diagnostic delay. Most patients had interactions with many healthcare professionals who did not recognise or believe the symptoms being reported, which in turn drives a thirst for knowledge at diagnosis and shapes subsequent attitudes towards doctors (6). Sibeoni et al. (7) also reveal the psychosocial elements of a delayed diagnosis of acromegaly and suggest that endocrinologists should be involved in addressing the psychological impact of the condition together with the support of mental wellbeing.

A study with a focus group of six acromegalic patients to explore patient perceptions of disease impact presented a wide variety of causes for a reduced quality of life in patients with acromegaly (26). Some issues discussed in the focus group correlate with those reported by patients in our study. These include fatigue, mental health problems and worries about fertility (26). These issues are not covered in available disease-specific quality of life questionnaires. In their study, they advise clinicians to be more aware of these problems in order that appropriate support may be provided (26). It is clear that patients focus initially on the immediate treatment and resolution of symptoms such as fatigue, headaches and joint pain, but pay little attention to the potential long-term implications or need for future multimodal therapy (6). When and how patients should receive this information and how they will process it is not clear. However, our study and that of Gurel et al. (6) suggest this is most likely to have an impact when discussed in a patient-to-patient forum.

Despite a clear benefit from a multidisciplinary team approach to the management of pituitary disease $(9,27)$, patients find the experience intimidating. Although not all centres will adopt a joint MDT clinic with multiple clinicians; in our practice, we find it beneficial to facilitate discussion between the patient, endocrinologist, surgeon and radiation oncologist to explore all treatment options. Prior contact from a specialist nurse or written information detailing the team members and their roles may help ease some anxiety and improve subsequent treatment adherence and overall satisfaction (11).

Overall, despite increasing awareness of acromegaly and a recent reduction in the delay to diagnosis (4), most patients are diagnosed in non-specialist centres with limited access to specific support resources. There is a need for clear signposting to up-to-date online resources that patients feel is relevant to them both factually and geographically. Our study demonstrates this should occur prior to attendance at the specialist tertiary centre together with a need for supporting education on acromegaly in primary and secondary care.

\section{Limitations}

This study involves patients from a single centre and results drawn from the study may not apply to other patient groups and/or internationally. Nevertheless, conclusions drawn from this study may still be relevant for other centres to better appreciate the needs of patients with acromegaly. In any qualitative study, the interviewer's ideas and assumptions can bias the outcome; however, the semi-structured format and open questions should have allowed the patients to speak freely about their experience and concerns.

\section{Conclusion}

The study demonstrates some of the challenges faced by patients with acromegaly and gives us an insight into the knowledge of their condition and what information and support they require. It highlights a need for better communication with patients as well as guidance for online searching. There is a clear need for the provision of information at the point of initial diagnosis in whatever care setting that may be made. The wider education and dissemination of appropriate online resources will improve subsequent health care provider-patient communication and ultimately improve treatment satisfaction and quality of life.

\section{Declaration of interest}

The authors declare that there is no conflict of interest that could be perceived as prejudicing the impartiality of the research reported.

\section{Funding}

This work did not receive any specific grant from any funding agency in the public, commercial or not-for-profit sector.

\section{Author contribution statement}

$H Y \vee P, C H$ and $A L$ devised the study. $H Y \vee P$ undertook interviews. $H Y \vee P, C H$ and J S D reviewed thematic analysis. All authors contributed to manuscript preparation and review.
This work is licensed under a Creative Commons Attribution-NonCommercial 4.0 International License. ded from Bioscientifica.com at 04/26/2023 12:42:39PM 


\section{References}

1 Colao A, Grasso LFS, Giustina A, Melmed S, Chanson P, Pereira AM \& Pivonello R. Acromegaly. Nature Reviews: Disease Primers 2019520. (https://doi.org/10.1038/s41572-019-0071-6)

2 AlDallal S. Acromegaly: a challenging condition to diagnose. International Journal of General Medicine 201811 337-343. (https:// doi.org/10.2147/IJGM.S169611)

3 Abreu A, Tovar AP, Castellanos R, Valenzuela A, Giraldo CMG, Pinedo AC, Guerrero DP, Barrera CAB, Franco HI, Ribeiro-Oliveira Jr $A$, et al. Challenges in the diagnosis and management of acromegaly: a focus on comorbidities. Pituitary 201619 448-457. (https://doi. org/10.1007/s11102-016-0725-2)

4 Caron P, Brue T, Raverot G, Tabarin A, Cailleux A, Delemer B, Renoult PP, Houchard A, Elaraki F \& Chanson P. Signs and symptoms of acromegaly at diagnosis: the physician's and the patient's perspectives in the ACRO-POLIS study. Endocrine 201963 120-129. (https://doi.org/10.1007/s12020-018-1764-4)

5 Dekkers OM, Biermasz NR, Pereira AM, Romijn JA \& Vandenbroucke JP. Mortality in acromegaly: a metaanalysis. Journal of Clinical Endocrinology and Metabolism 200893 61-67. (https://doi. org/10.1210/jc.2007-1191)

6 Gurel MH, Bruening PR, Rhodes C \& Lomax KG. Patient perspectives on the impact of acromegaly: results from individual and group interviews. Patient Preference and Adherence 20148 53-62. (https:// doi.org/10.2147/PPA.S56740)

7 Sibeoni J, Manolios E, Verneuil L, Chanson P \& Revah-Levy A. Patients' perspectives on acromegaly diagnostic delay: a qualitative study. European Journal of Endocrinology 2019180 339-352. (https:// doi.org/10.1530/EJE-18-0925)

8 Raverot G, Burman P, McCormack A, Heaney A, Petersenn S, Popovic V, Trouillas J, Dekkers OM \& European Society of Endocrinology. European Society of Endocrinology Clinical Practice Guidelines for the management of aggressive pituitary tumours and carcinomas. European Journal of Endocrinology 2018178 G1-G24. (https://doi.org/10.1530/EJE-17-0796)

9 McLaughlin N, Laws ER, Oyesiku NM, Katznelson L \& Kelly DF. Pituitary centers of excellence. Neurosurgery 201271 916-924; discussion 924-916. (https://doi.org/10.1227/NEU.0b013e31826d5d06)

10 Dal J, Feldt-Rasmussen U, Andersen M, Kristensen LØ, Laurberg P, Pedersen L, Dekkers OM, Sorensen HT \& Jorgensen JO. Acromegaly incidence, prevalence, complications and long-term prognosis: a nationwide cohort study. European Journal of Endocrinology 2016175 181-190. (https://doi.org/10.1530/EJE-16-0117)

11 Plunkett C \& Barkan AL. The care continuum in acromegaly: how patients, nurses, and physicians can collaborate for successful treatment experiences. Patient Preference and Adherence 20159 1093-1099. (https://doi.org/10.2147/PPA.S84887)

12 Kennedy TJ \& Lingard LA. Making sense of grounded theory in medical education. Medical Education 200640 101-108. (https://doi. org/10.1111/j.1365-2929.2005.02378.x)

13 Strauss A \& Corbin J. Basics of Qualitative Research: Techniques and Procedures for Developing Grounded Theory. Thousand Oaks, CA, USA: Sage Publications, 1998.
14 Glaser BG \& Strauss AL. The Discovery of Grounded Theory: Strategies for Qualitative Research. Chicago, IL, USA: Aldine, 1967.

15 Chun Tie Y, Birks M \& Francis K. Grounded theory research: a design framework for novice researchers. SAGE Open Medicine 201972050312118822927 . (https://doi. org/10.1177/2050312118822927)

16 Chapman AL, Hadfield M \& Chapman CJ. Qualitative research in healthcare: an introduction to grounded theory using thematic analysis. Journal of the Royal College of Physicians of Edinburgh 201545 201-205. (https://doi.org/10.4997/JRCPE.2015.305)

17 Crowe AL, McKnight AJ \& McAneney H. Communication needs for individuals with rare diseases within and around the Healthcare System of Northern Ireland. Frontiers in Public Health 20197 236-236. (https://doi.org/10.3389/fpubh.2019.00236)

18 Stanarević Katavić S. Health information behaviour of rare disease patients: seeking, finding and sharing health information. Health Information and Libraries Journal 201936 341-356. (https://doi. org/10.1111/hir.12261)

19 Chen YY, Li CM, Liang JC \& Tsai CC. Health information obtained From the internet and changes in medical decision making: questionnaire development and cross-sectional survey. Journal of Medical Internet Research 201820 e47. (https://doi.org/10.2196/ jmir.9370)

20 Pauer F, Litzkendorf S, Göbel J, Storf H, Zeidler J \& Graf von der Schulenburg JM. Rare diseases on the internet: an assessment of the quality of online information. Journal of Medical Internet Research 201719 e23-e23. (https://doi.org/10.2196/jmir.7056)

21 Amann J, Rubinelli S \& Kreps G. Revisiting the concept of health literacy. The patient as information seeker and provider. European Health Psychologist 201517 286-290.

22 Polanco-Briceno S, Glass D \& Plunkett C. Communication practices and awareness of resources for acromegaly patients among endocrinologists. Patient Preference and Adherence 201610 2531-2541. (https://doi.org/10.2147/PPA.S119570)

23 Maragh-Bass AC, Appelson JR, Changoor NR, Davis WA, Haider AH \& Morris MA. Prioritizing qualitative research in surgery: a synthesis and analysis of publication trends. Surgery $20161601447-1455$ (https://doi.org/10.1016/j.surg.2016.06.026)

24 Gallo L, BHSc, Murphy J, Braga LH, Farrokhyar F \& Thoma A. Users' guide to the surgical literature: how to assess a qualitative study. Canadian Journal of Surgery 201861 208-214. (https://doi. org/10.1503/cjs.013117)

25 Geer EB, Sisco J, Adelman DT, Ludlam WH, Haviv A, Gelbaum D, Liu S, Mathias SD \& Shi L. Observed discordance between outcomes reported by acromegaly patients and their treating endocrinology medical provider. Pituitary 202023 140-148. (https://doi. org/10.1007/s11102-019-01013-2)

26 Andela CD, Niemeijer ND, Scharloo M, Tiemensma J, Kanagasabapathy S, Pereira AM, Kamminga NG, Kaptein AA \& Biermasz NR. Towards a better quality of life (QoL) for patients with pituitary diseases: results from a focus group study exploring QoL. Pituitary 201518 86-100. (https://doi.org/10.1007/s11102-0140561-1)

27 Bevan JS. Management of pituitary tumours. BMJ 1999318 1226-1227. (https://doi.org/10.1136/bmj.318.7193.1226)

Received in final form 17 August 2020

Accepted 10 September 2020

Accepted Manuscript published online 11 September 2020 https://ec.bioscientifica.com https://doi.org/10.1530/EC-20-0335 (c) 2020 The authors Published by Bioscientifica Ltd

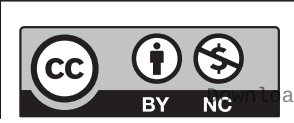

This work is licensed under a Creative Commons Attribution-NonCommercial 4.0 International License. ded from Bioscientifica.com at 04/26/2023 12:42:39PM 